\title{
Performance Analysis of Wireless Sensor Network Based on OPNET ${ }^{*}$
}

\author{
Yi Sun, Yue Sun, Peng Xu, Haocheng Liu \\ School of Electrical and Electronic Engineering, North China Electric Power University, Beijing, China \\ Email: sy@ncepu.edu.cn, timsunyue@126.com, xupeng0903@126.com
}

Received May 2013

\begin{abstract}
The wireless sensor network is a new hot spot in the field of wireless network. Compared with other network technology, wireless sensor network has less communication protocols, mainly including Wireless HART, ISA100.11a, IEEE1451, and ZigBee/IEEE802.15.4. IEEE802.15.4 was designed for short-range, low-complexity, low-power, lowrate and low-cost wireless network which provides two-way wireless communication technology [1]. In this paper, the performance of IEEE 802.15.4 is analyzed based on OPNET simulator. The simulation result indicates the influence of ACK mechanism and different network load on the system performance, i.e. end-to-end delay, packet reception ratio and throughout of node, which provides an important theoretical basis for the construction of actual network.
\end{abstract}

Keywords: Wireless Sensor Network; OPNET; IEEE802.15.4; Performance

\section{Introduction}

As wireless communication, integrated circuits, sensor and micro electromechanical system, i.e. MEMS, are rapidly developing, it is possible to the mass production of tiny sensor nodes with the functions of wireless communication, data acquisition and processing and collaboration [2]. Wireless sensor network is an intelligent private network composed of sensor nodes with specific functions which exchange information by self-organizing wireless communication and accomplish a specific function together [3]. IEEE802.15.4 agreement with low-rate aims at meeting the need of low-power and low-cost in the industrial automation, intelligent household, medical application, etc. Therefore, the research of the performance of IEEE802.15.4 is crucial for the design of wireless sensor network.

OPNET employs object modeling method and graphical editor, providing comprehensive simulation development environment for the communication network and modeling of a distributed system. Based on event driven mechanism and compared with the time driven, computational efficiency has been greatly improved [4]. OPNET provides three modeling mechanism: the bottom is the process model, implementing the algorithm agreement; the middle is the node model, employing process model to implement the corresponding equipment func-

*The work was supported by a grant from the National Science and Technology Major Project of China (No.2010ZX03006-005-01). tion; the top is the network model, employing node model to construct reality network topology structure. Three layer models completely correspond to the actual protocol, equipment and network, which fully reflect the network related features.

The purpose of this paper is to investigate the performance of IEEE802.15.4 in different network requirements. We use OPNET to analyze different scenarios, i.e. whether to adopt an ACK mechanism and different network load. According to the simulation results we analyze the performance of different parameters, including different mode and load condition. Based on the simulation we can get a suitable solution for the network performance.

The rest of this paper is arranged as following. A brief description of IEEE802.15.4 is given in Section 2. Section 3 introduces the selection of simulation topology. The simulation parameter configuration is given in Section 4. Simulating results are depicted and analyzed in Section 5 and then Section 6 gives the conclusion.

\section{The IEEE 802.15.4 MAC Protocol}

IEEE802.15/TG4 formulated the IEEE802.15.4 for lowrate wireless personal area network, i.e. LR-WPAN. The standard gives priority to low-power, low-rate and lowcost, aiming at providing unified standard for individuals or families. The characteristics of LR-WPAN network are similar to the sensor network, and it is regarded as 
sensor communication standard by many research institutions. The group devotes to the standard of the physical layer of WPAN network, i.e. PHY, and media access layer, i.e. MAC, aiming at providing communication standards to communicate with wireless communication device in the personal operating space, i.e. POS. POS generally refers to the scope of 10 meters near to users and users can be stationary or moving within this scope. The agreement is designed with the following features [5-7]:

- Data rates of $250 \mathrm{kbps}, 40 \mathrm{kbps}$ and $200 \mathrm{kbps}$.

- Support network topology: the star and peer to peer.

- Two addressing modes: 16-bit short and 64-bit IEEE addressing.

- Support optional time slot allocation.

- 16 channels in the $2.45 \mathrm{GHz}$ ISM band, 10 channels in the $915 \mathrm{MHz}$ ISM band and one channel in the 868 MHz band.

- Support carrier sense multiple access with collision avoidance, CSMA-CA.

- Handshake protocol for transfer reliability.

- Power management to ensure low power consumption.

- Support the link quality indicator.

The architecture of IEEE802.15.4 is shown in Figure

1. The structure includes the physical layer, the network layer and the application layer. The physical layer contains the control mechanism of the radio frequency transceiver along with its low-level and the access mechanism that the MAC sub-layer provides access to the physical channel. The network layer provides the network configuration and message routing. The application layer provides predetermined functions of equipment. Of these, the physical and medium access control layers have completed the standardized.

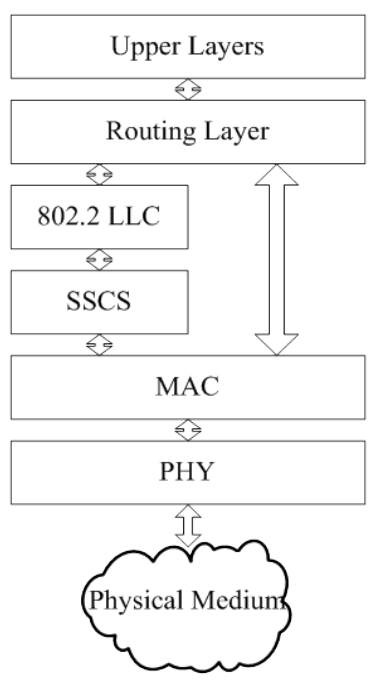

Figure 1. 802.15.4 System structure.

\section{The Selection of Simulation Topology}

In different application environment, IEEE802.15.4 provides two types of network topology: the star and peer to peer topology [7-9]. In this paper, mesh network and a cluster of tree network which is based on a peer to peer topology, as Figures $\mathbf{2}$ and $\mathbf{3}$ show, are used as the simulation scenarios. Although in practice the topology that a specific network adopt may differ, this two topologies are two of the most basic situation and have enough been used in our experiment to conclude some basic properties of IEEE802.15.4. In a mesh network, there is only one PAN coordinator. It allows any device to make message route other device in multiple hops and can provide reliable multi-path routing. Cluster tree network is a specific peer-to-peer network. Most of the equipment in the network is FFD, and RFD can only be connected to the end node on a brand. Any FFD can act as a coordinator which coordinate other devices and provide synchronization services. But at the same time there can be only one FFD as a coordinator in the entire network.

\section{Build Simulation Scenario}

The following three metrics are defined to measure the performance of IEEE 802.15.4.

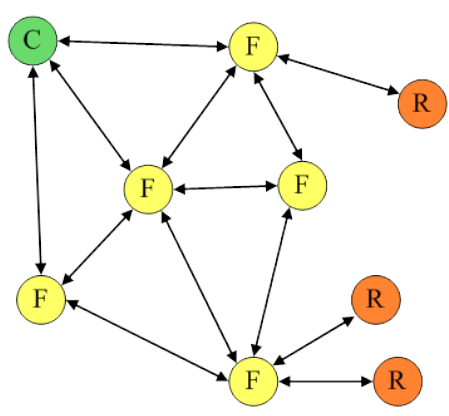

Figure 2. Mesh typology.

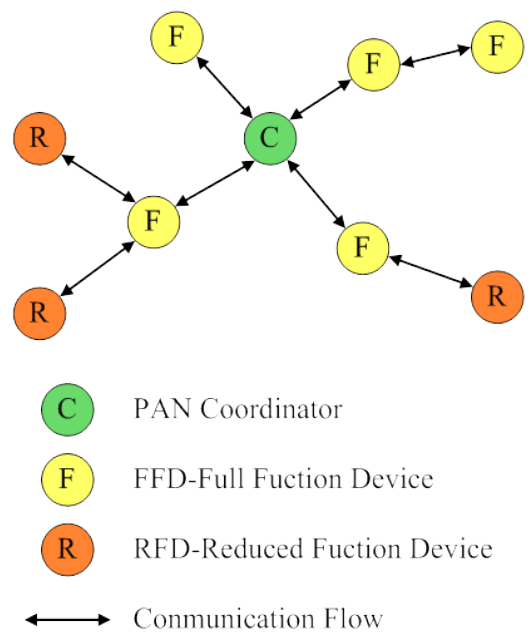

Figure 3. Cluster tree network. 
Throughout of the node: the number of forwarding data bits of each node in the network.

Delay: the needed time of a packet transmitted from one device to another in the network, including transmission delay, propagation delay, processing delay and queuing delay.

Packet reception ratio: the ratio of all sent packets to all packets successfully received in MAC layer of the coordinator.

A simulation scenario is built to test the performance. In this scenario, 15 nodes are distributed randomly in a $50 \times 50 \mathrm{~m}^{2}$ area. The source packets obey Poisson distribution. The message of application layer is 1024 bits. All the wireless transmission models are based on tworay-ground. What's more, the direct propagation path and the ground reflection path are both considered in the experiment, so it's more accurate than Free-Space model. The value of BO (Beacon Order) and SO (Superframe Order) are equal in the model, and there's no inactive part in Superframe.

The simulation scenario parameters are listed in Table 1.

\section{Experiment and Simulation Analysis}

Two experiments are designed to investigate the performance of IEEE 802.15.4.

Experiment 1: The effects of ACK (ACKnowledge Character) on the system performance.

This experiment is designed to compare the change of packet reception ratio and delay between two scenarios: one with ACK, and another without. This will provide a theoretical basis for that whether the ACK is needed when building a network. When a coordinator wishes to transfer data to a device in a beacon-enabled network, it indicates in the network beacon that the data message is pending. The device periodically listens to the network beacon, and if a message is pending, transmits a MAC command requesting this data, using slotted CSMA-CA. The device acknowledged the successful reception of the data by transmitting an acknowledgement frame. The process is shown in Figure 4, and the experiment results are shown in Figures 5 and $\mathbf{6}$.

Table 1. Parameters in Simulation Scenario [10-11].

\begin{tabular}{cc}
\hline Parameters & Values \\
\hline packet size (bit) & 1024 \\
Min backoff exponent & 3 \\
channel sensing duration (s) & 0.1 \\
packet reception-power threshold (W) & 0.5 \\
traffic type & poisson \\
routing protocol & AODV \\
simulation time (s) & 1200 \\
\hline
\end{tabular}

It can be seen that when packet interval time is less than $0.8 \mathrm{~s}$, ACK has little effect on the packet reception ratio, while the delay is much larger than the non-ACK

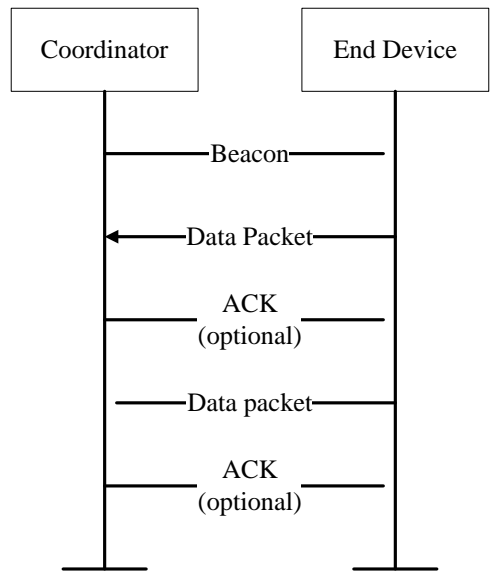

Figure 4. Pending Data from Coordinator to Device at Beacon-enabled Model.

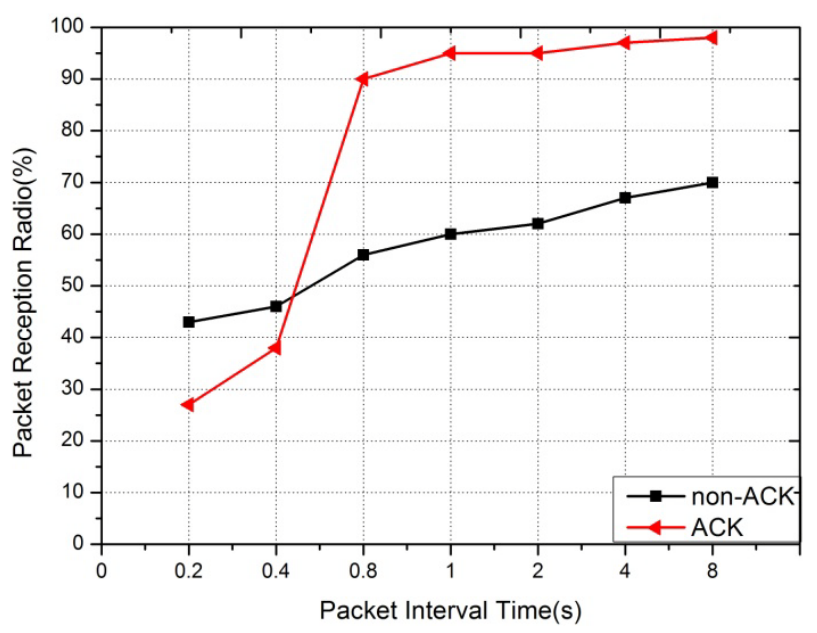

Figure 5. Influence of ACK on Reception Ratio.

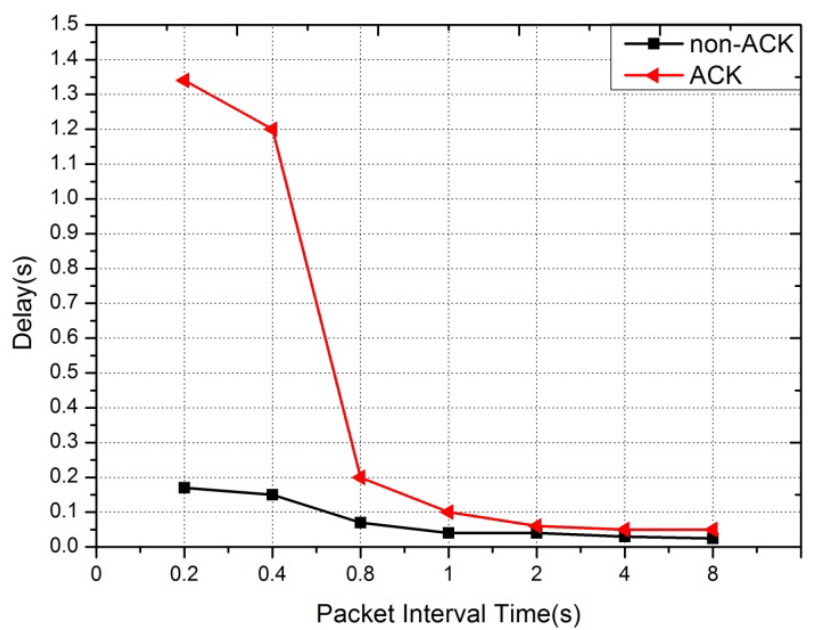

Figure 6. Influence of ACK on Delay. 
network. Once the interval time is larger than $0.8 \mathrm{~s}$, the packet reception ratio of scenario with ACK is 30 percent higher than the non-ACK one, while the delay between two scenarios are nearly the same. The reason is that when the sending interval time is very small, there will be many packets in the network which are transmitted at the same time. That will cause a higher collision ratio so the packet reception ratio is very low, besides, the network with ACK has more packets to transmit, so its delay is much larger. As the increasing of sending interval time, the probability of data collision is becoming lower, so that the efficiency of ACK is reflected. Therefore, ACK is appropriate for the network which packet interval time is larger. For the network with giant flow, ACK would have the opposite effect.

Experiment 2: The effect of node number on system performance.

This experiment is designed to trace the change of node throughout and delay in scenario with different node numbers, wishing to analyze how to deploy the density of nodes in different application scenario.

As shown in the Figures $\mathbf{7}$ and $\mathbf{8}$, when the node number is between 15 and 20, the throughput of node

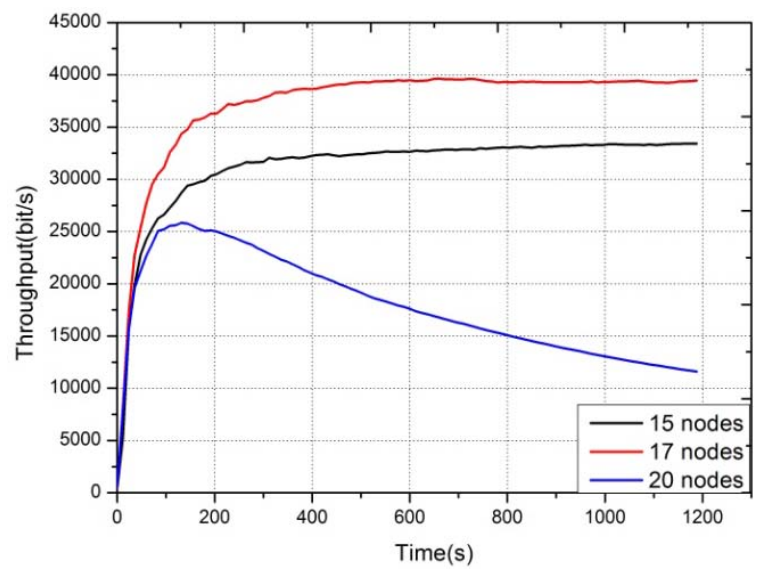

Figure 7. Throughout of Node.

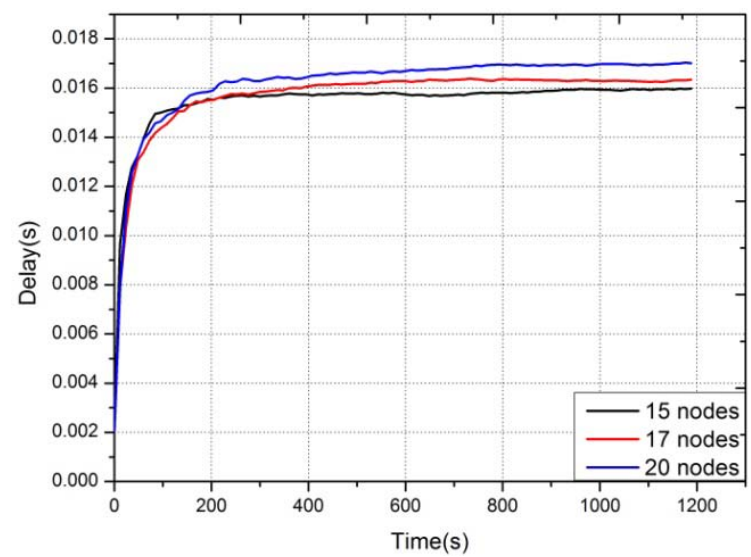

Figure 8. Average peer-to-peer delay. increases along with the node numbers. However, due to the network congestion, once the node number is larger than 20, the average throughout decreases. Since the increase of node numbers results in higher collision ratio, the delay also accordingly increases.

\section{Conclusion}

IEEE 802.15.4 was designed to meet the need for a lowcost and low-power wireless network. It has features such as low transmission rate, small communication range, easy to install, etc. In this paper, a scenario is built based on OPNET and two experiments are taken for investigating the performance of IEEE802.15.4. By using OPNET, we have simulated the influence of ACK on network performance in different packet intervals. According to the results, for the application environments with different requirement in time delay and reliability, the option of packet sending ratio and validation mechanism is flexible. In addition, by simulating the influence on the system throughput and delay in different network load conditions, it proves that network throughput has a largest threshold, and with the increase of network scale, network could reach congestion and lead to the deterioration of the network performance. Therefore, we should comprehensively investigate the network load impact on system performance when forming a network.

\section{REFERENCES}

[1] L. Xu, Z. D. Chen and C. Huang, "The Application of Game Theory in Wireless Sensor Network," Science Press, 2012.

[2] J. X. Liang, "Research on Energy Efficiency Technologies of MAC Protocols in WSN Based on IEEE 802.15.4," Master Thesis, Xidian University, Xi'an, 2010.

[3] R. Shorey, A. Ananda, M. C. Chan and W. T. Ooi, "Mobile Wireless and Sensor Networks Technology Application and Future Directions,” China Machine Press, 2010.

[4] W. B. Wang and J. W. Zhang, "OPNET Modeler and Simulation,” Posts \& Telecom Press, 2003.

[5] M Gribaudo, D Manini, A Nordio and C. F. Chiasserini, "Analysis of IEEE 802.15.4 Sensor Networks for Event Detection,” IEEE Global Telecommunications Conference, 2009, pp. 1-6.

[6] B Xia, Q Fu, D Li and L. Zhang. "Performance Evaluation and Channel Modeling," 16th Asia-Pacific Conference on Communications, 2010, pp. 497-502.

[7] Wireless Medium Access Control (MAC) and Physical Layer (PHY) Specifications for Low Rate Wireless Personal Area Networks (LRWPANs), IEEE Std. 802.15.4, 2006.

[8] P. D. Marco, P. Park, C. Fischione and K. H. Johansson, “Analytical Modelling of IEEE 802.15.4 for Multi-hop Networks with Heterogeneous Traffic and Hidden Terminals," IEEE Global Telecommunications Conference, 
2010, pp. 1-6.

[9] M. Gribaudo, D. Manini, A. Nordio and C. F. Chiasserini, “Transient Analysis of IEEE 802.15.4 Sensor Networks," IEEE Transactions on Wireless Communications, April 2011, pp. 1165-1175.

[10] Y. Zhang, S. Wang, Z. Liu, W. Zhou and D. Liu, "Performance Analysis of Wireless Sensor Network Based on
NS-2,” IEEE International Conference on Systems and Informatics, 2012, pp. 1445-1448.

[11] C. Ramassamy, I. HFoucha and P. Hunel, "Impact of Transmission Range in 802.15.4 with Usual Routing Protocols," Wireless Communications and Mobile Computing Conference (IWCMC), 2012, pp. 728-733. 